\title{
EFFECT OF ORAL KETAMINE AS ADJUVANT FOR TREATMENT OF NEUROPATHIC PAIN IN CANCER PATIENTS
}

\author{
Santanu Kumar Dash¹, Shakya Mohanty2, Diganta Mohanty³ \\ ${ }_{1}^{1}$ Associate Professor, Department of Anaesthesiology, Critical Care and Pain Management, Hitech Medical College and Hospital, \\ Bhubaneswar. \\ 2Final Year Postgraduate Student, Department of Anaesthesiology, Critical Care and Pain Management, Hitech Medical College and \\ Hospital, Bhubaneswar. \\ ${ }_{3}^{3}$ Final Year Postgraduate Student, Department of Anaesthesiology, Critical Care and Pain Management, Hitech Medical College and \\ Hospital, Bhubaneswar.
}

\begin{abstract}
BACKGROUND
ABSTRACT

The study was done to evaluate the effect of oral ketamine when it is used as an adjuvant in 35 cancer patients (19 men and 16 women) experiencing neuropathic pain. The patients were already on maximally tolerable doses of morphine and pregabalin for neuropathic pain and had a pain score $>6$ on a $0-10$ scale. Oral ketamine $0.5 \mathrm{mg} / \mathrm{kg}$ thrice daily was added to their regular medication. Patients were instructed to maintain a pain diary to record their pain score and record any other adverse effects. A decrease of $\geq 3$ from initial score or a score of $\leq 3$ was considered to be adequate response. Nine patients experienced nausea, out of which two had vomiting and five developed loss of appetite. Twenty-three patients complained of drowsiness initially, but it gradually decreased over 2 - 3 weeks among twenty of them. 2 of them withdrew (one on the $8^{\text {th }}$ and the other on the $12^{\text {th }}$ day) citing excessive sedation. 30 out of 35 patients experienced adequate pain relief. There were no complaints of visual or auditory hallucinations from any of the patients. The above study suggests that low-dose oral ketamine is potent and helpful in management of intractable neuropathic pain in cancer patients; however, we have to be observant regarding its side effects.
\end{abstract}

\section{MATERIALS AND METHODS}

This is a prospective observational study conducted by the Department of Anaesthesiology, Critical Care and Pain Management along with Department of Oncology of Hi-Tech Medical College and Hospital in the period from May 2016 - June 2017. After obtaining Institutional Ethical Committee clearance, we proceeded with the study. Cancer patients diagnosed with neuropathic pain based on clinical criteria were enrolled for the study.

\section{RESULTS}

In this study, 35 patients (19 men, 16 women) with neuropathic pain were included (Table 1 and 2). The mean average pain score prior to treatment was $8.03 \pm 0.68$ (Table 4). Twenty out of thirty-five patients had remarkable pain relief by the $10^{\text {th }}$ day. The mean NRS reduced to $5.06 \pm 1.43(\mathrm{p}<0.001)$ by the $10^{\text {th }}$ day of intervention. Thirty out of thirty-five patients had a decrease of more than 3 in NRS over the study period. The pain scores of these 30 patients varied between $3.0 \pm 0.78$ and $4.83 \pm 1.23$ over the next three weeks, i.e. till the end of the study.

\section{CONCLUSION}

In the above study, it can be seen that oral ketamine in low doses has been effective in treatment of neuropathic pain in cancer patients. The adverse effects of parenteral ketamine are much lesser when administered orally. Hence, the positive outcome lays the foundation for larger studies, which can help in setting up guidelines regarding indications for ketamine as an adjuvant to analgesic regimens.

\section{KEYWORDS}

Neuropathic Pain, Oral Ketamine, Cancer Pain, Opioid.

HOW TO CITE THIS ARTICLE: Dash SK, Mohanty S, Mohanty D. Effect of oral ketamine as adjuvant for treatment of neuropathic pain in cancer patients. J. Evolution Med. Dent. Sci. 2018;7(07):895-900, DOI: 10.14260/jemds/2018/204

\section{BACKGROUND}

Neuropathic pain is one of the most difficult and challenging aspects for a pain physician, because of its incomplete relief despite multiple medication and interventional procedures. ${ }^{1}$

'Financial or Other Competing Interest': None.

Submission 05-01-2017, Peer Review 01-02-2018,

Acceptance 07-02-2018, Published 12-02-2018.

Corresponding Author:

Dr. Shakya Mohanty,

Department of Anaesthesiology,

Hitech Medical College and Hospital,

Pandra, Rasulgarh,

Bhubaneswar-751025, Odisha.

E-mail: mohantyshakya@gmail.com

DOI: $10.14260 /$ jemds/2018/204
It can be described with various types of symptoms like sensory disturbances (Pain in area of sensory deficit, burning sensation), shooting pain, allodynia, hyperalgesia and dysaesthesias. Ketamine is an N-methyl D-aspartate (NMDA) receptor antagonist, which is routinely used as an anaesthetic agent. But in sub-anaesthetic doses (oral and parenteral), it is known to be a potent analgesic. ${ }^{2}$ It has been reported to be effective for reduction of pain in postherpetic neuralgia, glossopharyngeal neuralgia, phantom limb pain, central pain syndrome and cancer pain. ${ }^{2-18}$ There has not been much research regarding the use of oral ketamine examining its efficacy and adverse effect profile. In this study, we will try to assess the effect of oral ketamine as an adjuvant to oral morphine and oral pregabalin with respect to its efficacy and safety in cancer patients with neuropathic pain. 


\section{MATERIALS AND METHODS}

This prospective, observational study was conducted by the Department of Anaesthesiology, Critical Care and Pain Management along with Department of Oncology of Hi-Tech Medical College and Hospital in the period from May 2016 June 2017. After obtaining Institutional Ethical Committee clearance, we proceeded with the study. Cancer patients diagnosed with neuropathic pain based on clinical criteria were enrolled for the study. The sample size was based on the number of such cases attending pain clinic OPD. Taking into consideration the minimum sample size to be 30 along with a $10 \%$ dropout rate, we enrolled 35 patients for the study. The inclusion criteria was patient must be able to ambulate, able to take drugs orally, Karnofsky score $>60$, already on maximum tolerable dose of morphine and pregabalin and a pain score of $>6$ on a scale of $0-10$ in numerical rating scale (NRS). Patients who did not give consent were not able to take drugs orally, had increased intraocular/ intracranial pressure, any history of visual or auditory hallucinations and vertigo were excluded from the study. Patients or their attendants who would not be able to report or collect medication as per study design were also excluded.

The patients reporting for pain management were treated according to the World Health Organisation's (WHO) threestep ladder approach guidelines. Cancer patients who were diagnosed to have neuropathic pain were administered oral morphine (30 - $120 \mathrm{mg}$ qid) along with an anticonvulsant (oral pregabalin 75 - $150 \mathrm{mg}$ bd) and other analgesics. Doses were modified according to pain relief and dose limiting side effects.

Patients enrolled for the study completed a form with their demographic details and their current medications. Then they were briefed about the Brief Pain Inventory in Hindi (BPI-H). ${ }^{19}$ It records the maximum, minimum and average pain score on a 0 - 10 scale for a week. It also records how pain has affected various aspects of the patient's life. The scores were recorded according to the scale: sedation score 20 (0: alert, 1: drowsy, 2: sleepy but arousable, 3: unarousable) and vomiting score 21 (0: no nausea, 1: nausea alone, 2: one episode of emesis, 3: two or more episodes of emesis requiring anti-emetics).

The injectable form of ketamine $(50 \mathrm{mg} / \mathrm{mL})$ was mixed with a sweet syrup (sugar solution) till a concentration of 10 $\mathrm{mg} / \mathrm{mL}$ was obtained. This was prescribed to all the patients in the dose of $0.5 \mathrm{mg} / \mathrm{kg}$ thrice daily along with their present drug regime. This dosage was derived from previously published reports $2,10,22$ and pharmacokinetics of ketamine and its metabolite norketamine. ${ }^{10,23-27}$

Patients and their attendants were taught to maintain a pain diary where they were supposed to record maximum and minimum pain score, sedation score, vomiting score and any other side effects (hallucinations, dizziness, vivid dreams, floating sensation, delirium) every evening. Patients were admitted in the hospital for the first 5 - 7 days, so as to make them acquainted with the timings of medication and how to maintain the pain diary. The record for each patient was maintained for 30 days. For patients who were unable to report on a regular basis, their relatives were asked to report with the pain diary and collect the medication. The pain score was rated as average pain score of the day on a scale of $0-10$. A decrease of 3 or more from the baseline score in NRS or a score $\leq 3$ was considered to be adequate response. In case of any side effect, patient was administered appropriate medication for it. If any patient was unable to cope with the new medication for any reason, he/ she was excluded from the study. The data collected from patients who discontinued were not included in statistical analysis of the study.

\section{Statistical Analysis}

The data was evaluated by non-parametric statistical analysis using SPSS 20.0 software. Friedman test by ranks was used to calculate significant changes in NRS, vomit and sedation scores. $\mathrm{P} \leq 0.05$ was considered to be significant. Data were expressed as the mean \pm SD and median and IQR.

\section{RESULTS}

In this study, 35 patients (19 men, 16 women) with neuropathic pain were included (Table 1 and 2). The mean average pain score prior to treatment was $8.03 \pm 0.68$ (Table 4). Twenty out of thirty five patients had remarkable pain relief by the $10^{\text {th }}$ day. The mean NRS reduced to $5.06 \pm 1.43$ (p $<0.001$ ) by the $10^{\text {th }}$ day of intervention. Thirty out of thirty five patients had a decrease of more than 3 in NRS over the study period. The pain scores of these 30 patients varied between $3.0 \pm 0.78$ to $4.83 \pm 1.23$ over the next three weeks, i.e. till the end of the study.

Nine patients experienced nausea, out of which two had vomiting and five had complaints of loss of appetite (Table 5). The incidence of nausea after starting oral ketamine was statistically insignificant ( $p>0.50)$. Sedation/ drowsiness was reported by twenty three of them (Table 6) and it was statistically significant $(\mathrm{p}<0.01)$, but it gradually decreased over a period of two to three weeks despite continuation of medication in twenty one of those twenty three patients. Two of these, 23 patients withdrew (one on the 8th day and the other on the 12th day) citing excessive sedation. Thirty three patients completed the study. None of the patients complained of visual or auditory hallucinations, vertigo and delirium. There was no attempt made to reduce dosage of any current pain medication during the course of study. All the patients who had pain relief (30 out of 33 who completed study) continued with oral ketamine. Alternative medication was tried for the patient who did not have successful pain relief.

\begin{tabular}{|c|c|c|c|c|c|}
\hline Patient & $\begin{array}{c}\text { Age/ } \\
\text { Sex }\end{array}$ & $\begin{array}{c}\text { Cancer } \\
\text { Site }\end{array}$ & $\begin{array}{c}\text { Site of } \\
\text { Pain }\end{array}$ & $\begin{array}{c}\text { Duration } \\
\text { of Pain } \\
\text { (Months) }\end{array}$ & $\begin{array}{c}\text { Nature } \\
\text { of Pain }\end{array}$ \\
\hline 1 & $54 / \mathrm{M}$ & Tongue & $\begin{array}{c}\text { Cheeks } \\
\text { and ear }\end{array}$ & 7 & $\begin{array}{c}\text { Sharp, } \\
\text { shooting }\end{array}$ \\
\hline 2 & $51 / \mathrm{M}$ & Stomach & $\begin{array}{c}\text { Chest and } \\
\text { upper } \\
\text { abdomen } \\
\text { pain }\end{array}$ & 3 & $\begin{array}{c}\text { Burning, } \\
\text { shooting }\end{array}$ \\
\hline 3 & $50 / \mathrm{M}$ & Larynx & Neck & 14 & $\begin{array}{c}\text { Burning, } \\
\text { sharp }\end{array}$ \\
\hline 4 & $46 / \mathrm{M}$ & Alveolus & Chest pain & 4 & $\begin{array}{c}\text { Allodynia, } \\
\text { burning }\end{array}$ \\
\hline 5 & $52 / \mathrm{F}$ & Breast & Upper limb & 3 & $\begin{array}{c}\text { Shooting, } \\
\text { numbness }\end{array}$ \\
\hline 6 & $44 / \mathrm{M}$ & Testicular & $\begin{array}{c}\text { Low back } \\
\text { and thighs }\end{array}$ & 4 & $\begin{array}{c}\text { Shooting, } \\
\text { allodynia }\end{array}$ \\
\hline 7 & $61 / \mathrm{M}$ & Tongue & Cheeks & 7 & $\begin{array}{c}\text { Sharp, } \\
\text { shooting }\end{array}$ \\
\hline 8 & $64 / \mathrm{F}$ & Breast & Chest and & 11 & Shooting, \\
\hline
\end{tabular}




\begin{tabular}{|c|c|c|c|c|c|}
\hline & & & upper limb & & throbbing \\
\hline 9 & $34 / F$ & Tongue & $\begin{array}{c}\text { Cheeks } \\
\text { and ear }\end{array}$ & 7 & $\begin{array}{c}\begin{array}{c}\text { Sharp, } \\
\text { shooting }\end{array} \\
\end{array}$ \\
\hline 10 & $54 / F$ & Oesophagus & Chest pain & 2 & $\begin{array}{l}\text { Throbbing, } \\
\text { shooting }\end{array}$ \\
\hline 11 & $57 / \mathrm{M}$ & Prostate & Back pain & 4 & Shooting \\
\hline 12 & $54 / F$ & Larynx & $\begin{array}{l}\text { Neck and } \\
\text { cheeks }\end{array}$ & 6 & $\begin{array}{l}\text { Burning, } \\
\text { electric }\end{array}$ \\
\hline 13 & $64 / \mathrm{M}$ & Prostate & Low back & 3 & Shooting \\
\hline 14 & $56 / \mathrm{F}$ & Breast & Upper limb & 16 & $\begin{array}{c}\text { Shooting, } \\
\text { numbness }\end{array}$ \\
\hline 15 & $30 / \mathrm{M}$ & Tongue & Ear & 6 & $\begin{array}{l}\text { Shooting, } \\
\text { electric }\end{array}$ \\
\hline 16 & $43 / F$ & $\begin{array}{c}\text { Gum } \\
\text { mucosa }\end{array}$ & $\begin{array}{l}\text { Ear and } \\
\text { cheeks }\end{array}$ & 8 & $\begin{array}{c}\text { Sharp, } \\
\text { shooting }\end{array}$ \\
\hline 17 & $49 / \mathrm{M}$ & Testicular & $\begin{array}{c}\text { Low back } \\
\text { and thighs } \\
\end{array}$ & 7 & $\begin{array}{l}\text { Shooting, } \\
\text { allodynia }\end{array}$ \\
\hline 18 & $53 / \mathrm{M}$ & Tongue & $\begin{array}{c}\text { Cheeks } \\
\text { and ear }\end{array}$ & 5 & $\begin{array}{c}\text { Sharp, } \\
\text { shooting }\end{array}$ \\
\hline 19 & $56 / F$ & Breast & Upper limb & 8 & $\begin{array}{l}\text { Shooting, } \\
\text { numbness }\end{array}$ \\
\hline 20 & $46 / \mathrm{M}$ & Prostate & Back pain & 3 & Shooting \\
\hline 21 & $59 / \mathrm{F}$ & $\begin{array}{c}\text { Gum } \\
\text { mucosa }\end{array}$ & $\begin{array}{l}\text { Ear and } \\
\text { cheeks }\end{array}$ & 9 & $\begin{array}{l}\text { Shooting, } \\
\text { throbbing }\end{array}$ \\
\hline 22 & $64 / \mathrm{M}$ & $\begin{array}{c}\text { Gum } \\
\text { mucosa }\end{array}$ & $\begin{array}{c}\text { Ear and } \\
\text { cheeks }\end{array}$ & 11 & $\begin{array}{c}\text { Sharp, } \\
\text { shooting }\end{array}$ \\
\hline 23 & $41 / \mathrm{M}$ & Tongue & Cheeks & 5 & $\begin{array}{l}\text { Electric, } \\
\text { shooting }\end{array}$ \\
\hline 24 & $64 / F$ & Breast & \begin{tabular}{|c|} 
Chest and \\
upper limb
\end{tabular} & 6 & $\begin{array}{l}\text { Shooting, } \\
\text { throbbing }\end{array}$ \\
\hline 25 & $64 / \mathrm{M}$ & Lungs & $\begin{array}{c}\text { Chest and } \\
\text { upper back } \\
\text { pain }\end{array}$ & 3 & $\begin{array}{l}\text { Allodynia, } \\
\text { burning, } \\
\text { shooting }\end{array}$ \\
\hline 26 & $51 / \mathrm{F}$ & Larynx & $\begin{array}{c}\text { Neck and } \\
\text { cheeks }\end{array}$ & 6 & $\begin{array}{l}\text { Burning, } \\
\text { electric }\end{array}$ \\
\hline 27 & $59 / \mathrm{M}$ & Tongue & $\begin{array}{l}\text { Cheeks } \\
\text { and ear }\end{array}$ & 14 & $\begin{array}{c}\text { Sharp, } \\
\text { shooting }\end{array}$ \\
\hline 28 & $67 / \mathrm{M}$ & Prostate & Low back & 5 & $\begin{array}{l}\text { Shooting, } \\
\text { electric }\end{array}$ \\
\hline 29 & $64 / F$ & Breast & $\begin{array}{c}\text { Chest and } \\
\text { upper limb }\end{array}$ & 8 & $\begin{array}{l}\text { Shooting, } \\
\text { throbbing }\end{array}$ \\
\hline 30 & $56 / F$ & Tongue & $\begin{array}{c}\text { Cheeks } \\
\text { and ear }\end{array}$ & 9 & $\begin{array}{l}\text { Sharp, } \\
\text { shooting }\end{array}$ \\
\hline 31 & $50 / \mathrm{M}$ & Prostate & Low back & 4 & $\begin{array}{l}\text { Shooting, } \\
\text { electric }\end{array}$ \\
\hline 32 & $45 / \mathrm{F}$ & Breast & Upper limb & 10 & $\begin{array}{l}\text { Shooting, } \\
\text { electric }\end{array}$ \\
\hline 33 & $57 / \mathrm{M}$ & Stomach & $\begin{array}{l}\text { Chest and } \\
\text { upper } \\
\text { abdomen } \\
\text { pain }\end{array}$ & 5 & $\begin{array}{l}\text { Burning, } \\
\text { shooting }\end{array}$ \\
\hline 34 & $59 / \mathrm{F}$ & $\begin{array}{c}\text { Gum } \\
\text { mucosa }\end{array}$ & Ear & 2 & $\begin{array}{c}\text { Sharp, } \\
\text { shooting }\end{array}$ \\
\hline 35 & $68 / \mathrm{F}$ & Breast & $\begin{array}{c}\text { Upper limb } \\
\text { and chest }\end{array}$ & 3 & $\begin{array}{l}\text { Shooting, } \\
\text { numbness }\end{array}$ \\
\hline
\end{tabular}

\begin{tabular}{|c|c|c|c|}
\hline \multicolumn{4}{|c|}{ Drug Doses (mg/day) } \\
\hline Patient & Morphine & Pregabalin & Ketamine \\
\hline 1 & 300 & 225 & 75 \\
\hline 2 & 360 & 225 & 75 \\
\hline 3 & 360 & 300 & 60 \\
\hline 4 & 300 & 225 & 90 \\
\hline 5 & 300 & 225 & 60 \\
\hline 6 & 270 & 150 & 60 \\
\hline 7 & 300 & 225 & 60 \\
\hline 8 & 210 & 150 & 60 \\
\hline 9 & 240 & 225 & 60 \\
\hline 10 & 240 & 150 & 45 \\
\hline 11 & 420 & 300 & 90 \\
\hline 12 & 300 & 225 & 75 \\
\hline 13 & 420 & 300 & 75 \\
\hline 14 & 270 & 150 & 60 \\
\hline 15 & 480 & 300 & 75 \\
\hline 16 & 270 & 150 & 75 \\
\hline 17 & 420 & 225 & 90 \\
\hline 18 & 360 & 225 & 75 \\
\hline 19 & 360 & 300 & 75 \\
\hline 20 & 300 & 150 & 75 \\
\hline 21 & 270 & 225 & 60 \\
\hline 22 & 420 & 225 & 60 \\
\hline 23 & 360 & 150 & 90 \\
\hline 24 & 240 & 150 & 75 \\
\hline 25 & 300 & 150 & 60 \\
\hline 26 & 210 & 225 & 75 \\
\hline 27 & 240 & 150 & 75 \\
\hline 28 & 360 & 300 & 75 \\
\hline 29 & 360 & 225 & 75 \\
\hline 30 & 420 & 150 & 60 \\
\hline 31 & 300 & 150 & 60 \\
\hline 32 & 270 & 225 & 75 \\
\hline 33 & 360 & 300 & 90 \\
\hline 34 & 360 & 225 & 60 \\
\hline 35 & 360 & 225 & 75 \\
\hline & able 3. Dru & ssage Score & \\
\hline
\end{tabular}

\begin{tabular}{|c|c|c|c|c|}
\hline \multicolumn{6}{|c|}{ Pain Score VNRS (0 - 10) } \\
\hline Patient & $\mathbf{0}^{\text {th }}$ day & $\mathbf{1 0}^{\text {th }}$ day & $\mathbf{2 0}^{\text {th }}$ day & 30th day $^{\text {th }}$ \\
\hline 1 & 8 & 4 & 2 & 2 \\
\hline 2 & 7 & 5 & 3 & 3 \\
\hline 3 & 8 & 6 & 5 & 3 \\
\hline 4 & 9 & 5 & 3 & 3 \\
\hline 5 & 8 & 7 & 4 & 4 \\
\hline 6 & 8 & 3 & 3 & 3 \\
\hline 7 & 7 & 5 & 3 & 3 \\
\hline 8 & 7 & 4 & 3 & 2 \\
\hline 9 & 8 & 4 & 5 & 3 \\
\hline 10 & 9 & 4 & 4 & 3 \\
\hline 11 & 9 & 7 & 3 & 2 \\
\hline 12 & 8 & 7 & 5 & 5 \\
\hline 13 & 8 & 5 & 3 & 4 \\
\hline 14 & 7 & 3 & 3 & 3 \\
\hline 15 & 8 & 7 & W & - \\
\hline 16 & 8 & 4 & 2 & 3 \\
\hline 17 & 7 & 4 & 3 & 3 \\
\hline 18 & 8 & 6 & 4 & 4 \\
\hline 19 & 8 & 4 & 4 & 3 \\
\hline
\end{tabular}

\begin{tabular}{|c|c|c|c|}
\hline Age Group & \multicolumn{2}{|c|}{ Sex } & Total \\
\hline & Male & Female & \\
\hline $30-39$ & 1 & 1 & 2 \\
\hline $40-49$ & 5 & 2 & 7 \\
\hline $50-59$ & 8 & 9 & 17 \\
\hline$>60$ & 5 & 4 & 9 \\
\hline Total & $\mathbf{1 9}$ & $\mathbf{1 6}$ & $\mathbf{3 5}$ \\
\hline \multicolumn{4}{|c|}{ Table 2. Age Distribution } \\
\hline
\end{tabular}




\begin{tabular}{|l|l|l|l|l|}
\hline 20 & 9 & 9 & 7 & 8 \\
\hline 21 & 8 & 5 & 4 & 2 \\
\hline 22 & 8 & 4 & 2 & 2 \\
\hline 23 & 9 & 7 & 5 & 4 \\
\hline 24 & 7 & 3 & 2 & 2 \\
\hline 25 & 9 & 5 & 5 & 4 \\
\hline 26 & 8 & 6 & 6 & 7 \\
\hline 27 & 7 & 5 & 3 & 3 \\
\hline 28 & 8 & 4 & 3 & 3 \\
\hline 29 & 8 & 5 & 3 & 2 \\
\hline 30 & 9 & 7 & 7 & 5 \\
\hline 31 & 8 & 4 & 4 & 3 \\
\hline 32 & 8 & 7 & 7 & 4 \\
\hline 33 & 7 & W & - & - \\
\hline 34 & 9 & 5 & 3 & 3 \\
\hline 35 & 8 & 4 & 3 & 2 \\
\hline \multicolumn{5}{|c|}{ Table 4. Patient's Score in VNRS } \\
\hline
\end{tabular}

\begin{tabular}{|c|c|c|c|c|}
\hline \multicolumn{5}{|c|}{ Vomiting score $(0-3)$} \\
\hline Patient & $0^{\text {th }}$ day & $10^{\text {th }}$ day & $20^{\text {th }}$ day & $30^{\text {th }}$ day \\
\hline 1 & 0 & 0 & 0 & 0 \\
\hline 2 & 0 & 0 & 0 & 0 \\
\hline 3 & 0 & 0 & 0 & 0 \\
\hline 4 & 0 & 0 & 0 & 0 \\
\hline 5 & 0 & 2 & 1 & 0 \\
\hline 6 & 0 & 0 & 0 & 0 \\
\hline 7 & 0 & 0 & 0 & 0 \\
\hline 8 & 0 & 1 & 1 & 0 \\
\hline 9 & 0 & 0 & 0 & 0 \\
\hline 10 & 0 & 0 & 0 & 0 \\
\hline 11 & 0 & 1 & 1 & 0 \\
\hline 12 & 0 & 0 & 0 & 0 \\
\hline 13 & 0 & 0 & 0 & 0 \\
\hline 14 & 0 & 0 & 0 & 0 \\
\hline 15 & 0 & 0 & W & - \\
\hline 16 & 0 & 1 & 1 & 1 \\
\hline 17 & 0 & 0 & 0 & 0 \\
\hline 18 & 0 & 0 & 0 & 0 \\
\hline 19 & 0 & 0 & 0 & 0 \\
\hline 20 & 0 & 0 & 0 & 0 \\
\hline 21 & 0 & 2 & 1 & 0 \\
\hline 22 & 0 & 0 & 0 & 0 \\
\hline 23 & 0 & 0 & 0 & 0 \\
\hline 24 & 0 & 1 & 1 & 0 \\
\hline 25 & 0 & 0 & 0 & 0 \\
\hline 26 & 0 & 0 & 0 & 0 \\
\hline 27 & 0 & 0 & 0 & 0 \\
\hline 28 & 0 & 0 & 0 & 0 \\
\hline 29 & 0 & 1 & 1 & 1 \\
\hline 30 & 0 & 0 & 0 & 0 \\
\hline 31 & 0 & 0 & 0 & 0 \\
\hline 32 & 0 & 1 & 0 & 0 \\
\hline 33 & 0 & $\mathrm{~W}$ & - & - \\
\hline 34 & 0 & 0 & 0 & 0 \\
\hline 35 & 0 & 1 & 1 & 0 \\
\hline \multicolumn{5}{|c|}{ Table 5. Patients vomiting score } \\
\hline
\end{tabular}

\begin{tabular}{|c|c|c|c|c|}
\hline 2 & 0 & 1 & 0 & 0 \\
\hline 3 & 0 & 1 & 1 & 0 \\
\hline 4 & 0 & 0 & 0 & 0 \\
\hline 5 & 0 & 1 & 0 & 0 \\
\hline 6 & 0 & 0 & 0 & 0 \\
\hline 7 & 0 & 2 & 1 & 0 \\
\hline 8 & 0 & 0 & 0 & 0 \\
\hline 9 & 0 & 1 & 1 & 1 \\
\hline 10 & 0 & 1 & 1 & 0 \\
\hline 11 & 0 & 1 & 0 & 0 \\
\hline 12 & 0 & 0 & 0 & 0 \\
\hline 13 & 0 & 1 & 0 & 0 \\
\hline 14 & 0 & 0 & 0 & 0 \\
\hline 15 & 0 & 2 & W & - \\
\hline 16 & 0 & 2 & 1 & 0 \\
\hline 17 & 0 & 1 & 0 & 0 \\
\hline 18 & 0 & 0 & 0 & 0 \\
\hline 19 & 0 & 1 & 1 & 0 \\
\hline 20 & 0 & 1 & 1 & 0 \\
\hline 21 & 0 & 0 & 0 & 0 \\
\hline 22 & 0 & 1 & 0 & 0 \\
\hline 23 & 0 & 0 & 0 & 0 \\
\hline 24 & 0 & 2 & 1 & 0 \\
\hline 25 & 0 & 1 & 0 & 0 \\
\hline 26 & 0 & 1 & 0 & 0 \\
\hline 27 & 0 & 0 & 0 & 0 \\
\hline 28 & 0 & 1 & 1 & 0 \\
\hline 29 & 0 & 0 & 0 & 0 \\
\hline 30 & 0 & 1 & 0 & 0 \\
\hline 31 & 0 & 0 & 0 & 0 \\
\hline 32 & 0 & 1 & 1 & 0 \\
\hline 33 & 0 & W & - & - \\
\hline 34 & 0 & 1 & 0 & 0 \\
\hline 35 & 0 & 0 & 0 & 0 \\
\hline
\end{tabular}

\begin{tabular}{|c|c|c|c|c|c|}
\hline & D-0 & D-10 & D-20 & D-30 & P value \\
\hline $\begin{array}{c}\text { Mean } \\
\text { Pain } \\
\text { Score }\end{array}$ & $0.63 \pm$ & $\begin{array}{c}5.06 \pm \\
1.43\end{array}$ & $\begin{array}{c}3.84 \pm \\
1.46\end{array}$ & $\begin{array}{c}3.36 \pm \\
1.41\end{array}$ & $<\mathbf{0 . 0 0 1}$ \\
\hline $\begin{array}{c}\text { Mean } \\
\text { Vomiting } \\
\text { Score }\end{array}$ & 0 & $\begin{array}{c}0.33 \pm \\
0.59\end{array}$ & $\begin{array}{c}0.24 \pm \\
0.43\end{array}$ & $\begin{array}{c}0.06 \pm \\
0.24\end{array}$ & $>0.50$ \\
\hline $\begin{array}{c}\text { Mean } \\
\text { Sedation } \\
\text { Score }\end{array}$ & 0 & $\begin{array}{c}0.72 \pm \\
0.62\end{array}$ & $\begin{array}{c}0.33 \pm \\
0.47\end{array}$ & $\begin{array}{c}0.03 \pm \\
0.17\end{array}$ & $<0.01$ \\
\hline \multicolumn{7}{|c|}{ Table 7. Mean \pm SD of VNRS, Vomiting } \\
and Sedation Scores \\
\hline
\end{tabular}

\begin{tabular}{|c|c|c|c|c|c|c|c|c|}
\hline & \multicolumn{2}{|c|}{ D-0 } & \multicolumn{2}{c|}{ D-10 } & \multicolumn{2}{c|}{ D-20 } & \multicolumn{2}{c|}{ D-30 } \\
\hline & Med & IQR & Med & IQR & Med & IQR & Med & IQR \\
\hline $\begin{array}{c}\text { Pain } \\
\text { score }\end{array}$ & 8 & 0.5 & 5 & 2 & 3 & 2 & 3 & 1.5 \\
\hline $\begin{array}{c}\text { Vomiting } \\
\text { score }\end{array}$ & 0 & 0 & 0 & 1 & 0 & 1 & 0 & 0 \\
\hline $\begin{array}{c}\text { Sedation } \\
\text { score }\end{array}$ & 0 & 0 & 1 & 1 & 0 & 1 & 0 & 0 \\
\hline
\end{tabular}

Table 8. Median and IQR of VNRS, Vomiting, and Sedation Scores

\section{DISCUSSION}

Thirty out of thirty five patients had remarkable pain relief following administration of low-dose oral ketamine. Neuropathic cancer pain is usually difficult to treat because many times there is incomplete pain relief, hence from a clinician's point of view it poses a therapeutic challenge. The 
use of a wide variety of drugs and several techniques of pain relief for this purpose is a proof by itself of how difficult and challenging this task is.

It has been observed that oral ketamine is effective in treatment of several cases of neuropathic pain. ${ }^{10,12,14,22}$ It is also known to be an effective adjuvant to opioids, has opioid sparing effect and also reduces development of opioid tolerance. ${ }^{10}$ Ketamine is being used in patients for pain relief (Neuropathic pain, postoperative pain, other types of chronic pain), but mainly in parenteral form. There are hardly any studies conducted to determine the benefit as well adverse effect profile of oral ketamine on a long-term basis in cancer patients with neuropathic pain.

Ketamine was developed in 1963 and was primarily used as a dissociative anaesthetic agent, but in sub-anaesthetic doses it is known to be a potent analgesic. ${ }^{2}$ It is mainly a noncompetitive NMDA receptor antagonist, but it also has action on various other receptors which might be contributing to its analgesic effect.28,29,30 These include interaction with Kappa opioid, monoaminergic and muscarinic receptors, voltage sensitive calcium channels and also local anaesthetic action comparable to lidocaine in high doses. ${ }^{2,3,31-34}$ However, its action on NMDA receptor might be one of the main mechanism for pain relief, because NMDA receptors are shown to play a major role in central sensitisation, neural plasticity, opioid tolerance and development of chronic neuropathic pain. $3,7,18,28,29,35-39$ A combination of NMDA receptor antagonist and opioid in animal models has shown to have a synergistic analgesic effect along with minimising the development of opioid tolerance and dependence. ${ }^{38}$

Ketamine undergoes biotransformation in liver with its major metabolite being norketamine. ${ }^{40}$ After oral administration bioavailability of ketamine is low, i.e. $16 \%{ }^{24}$ but plasma levels of norketamine is higher. ${ }^{24,41}$ The serum ketamine level required for analgesia is $150 \mathrm{ng} / \mathrm{mL}$, but after administering oral ketamine peak serum ketamine level is around $35-55 \mathrm{ng} / \mathrm{mL}$ (one-third of parenteral ketamine). ${ }^{24}$ As compared to ketamine, norketamine (major metabolite) is one-third to one-fifth potent as an anaesthetic ${ }^{42}$ and has analgesic properties too. ${ }^{24,43}$ Few studies point towards the possibility of effective dose of ketamine administered orally could be lesser than that of parenteral dose.10,24-27 Due to high first pass metabolism, serum norketamine levels are $2-3$ times greater after oral ketamine as compared to parenteral ketamine. ${ }^{24,27}$ The peak analgesic effect of oral ketamine corresponds with peak serum levels of norketamine. ${ }^{24}$ This shows that norketamine has a major role to play in analgesic effect after oral ketamine. ${ }^{24,27}$

Oral ketamine is considered to have a better adverse effect profile as compared to parenteral ketamine and our findings regarding its psychomimetic effects is similar to few of the previous studies.10,14,18,22 This reduced incidence of psychomimetic effect could be due to oral route and low-dose ketamine. None of the patients in our study were administered midazolam or haloperidol as a prophylactic measure for psychomimetic effects.

Sedation or drowsiness was a prominent side effect in our study. It was seen in majority of the patients for initial 2 - 3 weeks of starting medication. Gradually, it decreased without any development of tolerance to its pain-relieving effect. Low-dose ketamine in combination with opioids does not aggravate or contribute to opioid induced sedation. 44,45 The increased severity of drowsiness in this study could be due to simultaneous use of pregabalin, which is known to have drowsiness as an adverse effect along with ketamine. This combination could have had a synergistic effect. However, the sedation scores returned to baseline values after around 3 - 4 weeks of ketamine therapy suggesting tolerance to this adverse effect. Nausea and loss of appetite were two other side effects in this study. Because of less severity, they were statistically insignificant. However, the patient's quality of life might have been affected. But without any comparison with controls, we cannot determine if this adverse effect is associated with ketamine therapy.

\section{CONCLUSION}

In the above study, it can be seen that oral ketamine in low doses has been effective in treatment of neuropathic pain in cancer patients. The adverse effects of parenteral ketamine are much lesser when administered orally. Hence, the positive outcome lays the foundation for larger studies, which can help in setting up guidelines regarding indications for ketamine as an adjuvant to analgesic regimens.

\section{REFERENCES}

[1] Hanks GW, Portenoy RK, MacDonald N, et al. Difficult pain problems. In: Doyle P, Hanks GWC, MacDonald N. eds. Oxford textbook of palliative medicine. Oxford: Oxford University Press 1993:257-62.

[2] White P, Way WL, Trevor AJ. Ketamine-its pharmacology and therapeutic uses. Anaesthesiology 1982;56(2):119-36.

[3] Mercadente S. Ketamine in cancer pain: an up-date. Palliative Medicine 1996;10(3):225-30.

[4] Fallon M, Welsh J. The role of ketamine in pain control. European J Palliat Care 1996;3(4):143-6.

[5] Eide P, Stubhaug A, Breivit H. Continuous subcutaneous administration of the NMDA receptor antagonist ketamine in the treatment of post-herpetic neuralgia. Pain 1995;61(2):221-8.

[6] Eide PK, Jorum E, Stubhaug A, et al. Relief of postherpetic neuralgia with the NMDA receptor antagonist ketamine: a double- blind, cross-over comparison with morphine and placebo. Pain 1994;58(3):347-54.

[7] Clark JL, Kalan GE. Effective treatment of severe cancer pain of the head using low dose ketamine in an opioid tolerant patient. J Pain Symptom Manage 1995;10(4):310-4.

[8] Roytblat L, Korotkouchko A, Katz J, et al. Postoperative pain: the effect of low-dose ketamine in addition to general anaesthetic. Anaesthetic Analgesia 1993;77(6):1161-5.

[9] Schmid RL, Sandler AN, Katz J. Use and efficacy of lowdose ketamine in the management of acute postoperative pain: a review of current techniques and outcomes. Pain 1999;82(2):111-25.

[10] Fisher K, Hagen NA. Analgesic effect of oral ketamine in chronic neuropathic pain of spinal origin: a case report. J Pain Symptom Manage 1999;18(1):61-6.

[11] Fine PG. Low dose ketamine in the management of opioid non-responsive terminal cancer pain. JPain Symptom Manage 1999;17(4):296-300.

[12] Hoffmann V, Coppejans $\mathrm{H}$, Vercauteren $\mathrm{M}$, et al. Successful treatment of postherpetic neuralgia with oral ketamine. Clinical J Pain 1994;10(3):240-2. 
[13] Klepstad P, Borchgrevink PC. Four years' treatment with ketamine and a trial of dextromethorphan in a patient with severe post-herpetic neuralgia. Acta Anaesthesiol Scand 1997;41(3):422-6.

[14] Nikolajsen L, Hansen PO, Jensen TS. Oral ketamine therapy in the treatment of post-amputation stump pain. Acta Anaesthesiol Scand 1997;41(3):427-9.

[15] Stannard CF, Porter GE. Ketamine hydrochloride in the treatment of phantom limb pain. Pain 1993;54(2):227-30.

[16] Persson J, Axelsson G, Hallin RG, et al. Beneficial effects of ketamine in a chronic pain state with allodynia, possibly due to central sensitization. Pain 1995;60(2):217-22.

[17] Mathisen LC, Skjelbred P, Skoglund LA, et al. Effect of ketamine, an NMDA receptor inhibitor, in acute and chronic orofacial pain. Pain 1995;61(2):215-20.

[18] Lauretti GR, Lima ICPR, Reis MP, et al. Oral ketamine and transdermal nitroglycerine as analgesic adjuvants to oral morphine therapy for cancer pain management. Anesthesiology 1999;90(6):1528-33.

[19] Saxena A, Mendoza T, Cleeland CS. The assessment of cancer pain in north India: the validation of the Hindi Brief Pain Inventory--BPI-H. J Pain Symptom Manage 1999;17(1):27-41.

[20] Kelleher AA, Black A, Penman S, et al. Comparison of caudal bupivacaine and diamorphine with caudal bupivacaine alone for repair of hypospadias. $\mathrm{Br} \mathrm{J}$ Anaesth 1996;77(5):586-90.

[21] Sadhasivam S, Saxena A, Kathirvel S, et al. The safety and efficacy of prophylactic ondansetron in preventing PONV in patients undergoing MRM. Anesth Analg 1999;89(6):1340-5.

[22] Kannan TR, Saxena A, Bhatnagar S, et al. Oral ketamine as an adjuvant to oral morphine for neuropathic pain in Cancer patients. J Pain \& Symptom Management 2002;23(1):60-5.

[23] Domino EF, Zsigmond EK, Domino LE, et al. Plasma levels of ketamine and two of its metabolites in surgical patients using a gas chromatographic mass fragmentographic assay. Anesth Analg 1982;61(2):8792.

[24] Grant IS, Nimmo WS, Clements JA. Pharmacokinetics and analgesic effects of IM and oral ketamine. $\mathrm{Br} \mathrm{J}$ Anaesth 1981;53(8):805-10.

[25] Fisher K, Coderre TJ, Hagen NA. Targetting the NMDA receptor for chronic pain management. Pre-clinical animal studies, recent clinical experience and future research directions. J Pain Symptom Manage 2000;20(5):358-73.

[26] Broadley KE, Kurowska A, Tookman A. Ketamine injection used orally. Palliative Medicine 1996;10(3):247-50.

[27] Ebert B, Mikkelsen S, Thorkildsen C, et al. Norketamine, the main metabolite of ketamine, is a non-competitive NMDA receptor antagonist in the rat cortex and spinal cord. European J Pharmacology 1997;333(1):99-104.

[28] Luczack J, Dickenson AH, Kotlinske-Lemieszek A. The role of ketamine, an NMDA receptor antagonist, in the management of pain. Progress in Palliative Care 1995;3(4):127-34.
[29] Price DD, Mayer DJ, Mao J, et al. NMDA receptor antagonists and opioid receptor interactions as related to analgesia and tolerance. J Pain Symptom Manage 2000;19(Suppl 1):S7-S11.

[30] Rabben T, Skjelbred P, Oye I. Prolonged analgesic effect of ketamine, an NMDA receptor inhibitor in patients with chronic pain. J Pharmacology Experimental Therapeutics 1999;289(2):1060-6.

[31] Reich DL, Silvay G. Ketamine: an update on the first 25 years of clinical experience. Canadian J Anaesthesia 1989;36(2):186-97.

[32] Hirota K, Lambert DG. Ketamine: its mechanism(s) of action and unusual clinical uses. Brit J Anaesthesia 1996;77(4):441-4.

[33] Zhou ZS, Zhao ZQ. Ketamine blockage of both tetrodotoxin [TTX]-sensitive and TTX-resistant sodium channels of rat dorsal root ganglion neurons. Brain Res Bull 2000;52(5):427-33.

[34] Irifune M, Sato T, Kamata Y, et al. Evidence for GABA [A] receptor agonistic properties of ketamine: convulsive and anesthetic behavioral models in mice. Anesth Analg 2000;91(1):230-6.

[35] Portenoy RK. Evolving role of NMDA-receptor antagonists in analgesia. J Pain Symptom Manage 2000;19(Suppl 1):S1.

[36] Bennett GJ. Update on the neurophysiology of pain transmission and modulation: focus on the NMDA receptor. J Pain Symptom Manage 2000;19(Suppl 1):S2-S6.

[37] Bell RF. Low dose subcutaneous ketamine infusion and morphine tolerance. Pain 1999;83(1):101-3.

[38] Mao J, Price D, Caruso F, et al. Oral administration of dextromethorphan prevents the development of morphine tolerance and dependence in rats. Pain 1996;67(2-3):361-8.

[39] Adriaenssens G, Vermayen KM, Hoffman V, et al. Postoperative analgesia with i.v. patient controlled morphine: effect of adding ketamine. Brit J Anaesthetics 1999;83(3):393-6.

[40] Cohen ML, Chan SL, Way WL, et al. Distribution in brain and metabolism of ketamine in the rat after intravenous administration. Anesthesiology 1973;39(4):370-6.

[41] Clements JA, Nimmo WS. The pharmacokinetics and analgesic effect of ketamine in man. Br J Anaesth 1981;53(1):27-30.

[42] White PF, Johnston RR, Pudwill CR. Interaction of ketamine and halothane in rats. Anesthesiology 1975;42(2):179-86.

[43] Shimoyama M, Shimoyama N, Gorman AL, et al. Oral ketamine is antinociceptive in the rat formalin test: role of metabolite norketamine. Pain 1999;81(1-2):8593.

[44] Javery KB, Ussery TW, Steger HG, et al. Comparison of morphine and morphine with ketamine for postoperative analgesia. Can J Anaesth 1996;43(3):212-5.

[45] Stubhaug A, Breivik H, Eide PK, et al. Mapping of punctuate hyperalgesia around a surgical incision demonstrates that ketamine is a powerful suppressor of central sensitisation to pain following surgery. Acta Anaesthesiol Scand 1997;41(9):1124-32. 